Bio - grafía. Escritos sobre la Biología y su Enseñanza. ISSN 2027-1034

Edición Extraordinaria. p.p. 431- 440

Memorias del IX Encuentro Nacional de Experiencias en Enseñanza de la Biología y la Educación Ambiental. IV Congreso Nacional de Investigación en Enseñanza de la Biología.

\title{
CONOCIMIENTO DEL CONTENIDO EN FORMULACIÓN DE PREGUNTAS DE COMPETENCIAS ESPECÍFICAS EN LA PRUEBA SABER PRO PARA DOCENTES EN FORMACIÓN DE CIENCIAS NATURALES EN COLOMBIA.
}

\author{
Velásquez, Juan Nicolás \\ Rios, Christian Reynel \\ Martinez, Cristian Yasser \\ Mendoza, Luis Felipe
}

\section{RESUMEN:}

El presente documento aborda la forma en la cual están estructuradas las preguntas de la evaluación externa SABER PRO diseñada por el Instituto Colombiano para el Fomento de la Educación Superior (ICFES) para docentes en formación. En primera medida, se realiza una descripción general de las competencias que orientan dicha prueba, así como los núcleos y desempeños que dirigen los objetivos de estas; de esta manera se podrá evidenciar si se encuentra dentro de sus preocupaciones el estado del conocimiento del contenido en la evaluación de competencias específicas de docentes en formación. Como principal conclusión de este trabajo se puede afirmar que el conocimiento del contenido en las pruebas SABER PRO está ausente.

PALABRAS CLAVES: Conocimiento del Contenido, competencias específicas, formación docente, pruebas SABER PRO.

\section{ABSTRACT:}

This document addresses the way in which the SABER PRO external evaluation questions designed by the Colombian Institute for the Promotion of Higher Education (ICFES) for teachers in training are structured. In the first step, a general description of the competences that guide this test is made, as well as the nuclei and the performances that direct the objectives of these tests; in this way it can be evidenced if the content knowledge status in the evaluation of specific competences of teachers in training is within their concerns. The main conclusion of this work is that the knowledge of content in the SABER PRO tests is absent.

KEYWORDS: Knowledge of the Content, specific competences, teacher training, SABER PRO tests.

\section{INTRODUCCIÓN:}

Actualmente la evaluación de la educación superior en Colombia se encuentra dentro del modelo por competencias así que es pertinente iniciar dando las nociones básicas de este concepto y su aplicación en la evaluación. Competencia definida por Rey (1996), es la capacidad que desarrolla un sujeto para solucionar problemas de 


\section{Bio - grafía. Escritos sobre la Biología y su Enseñanza. ISSN 2027-1034}

Edición Extraordinaria. p.p. 431- 440

Memorias del IX Encuentro Nacional de Experiencias en Enseñanza de la Biología y la Educación Ambiental. IV Congreso Nacional de Investigación en Enseñanza de la Biología.

diversa índole, movilizando recursos propios y regulando el proceso en busca de alcanzar una meta. La apuesta formativa por el desarrollo de competencias en los estudiantes supone transformaciones en las instituciones en relación con los currículos, las estrategias didácticas y pedagógicas y las prácticas evaluativas, lo cual implica que el Ministerio de Educación, las universidades y las asociaciones de profesionales deben asumir investigaciones y generar reflexiones sobre la formación y evaluación por competencias. Esta tarea fue asumida inicialmente por el Instituto Colombiano para el Fomento de la Educación Superior (ICFES) y la Asociación Colombiana de Facultades de Educación (ASCOFADE), quienes "a partir de la revisión del proyecto Definition and Selection of Competencies (DeSeCo) y el Proyecto Tuning, en los cuales se seleccionan y definen las competencias en: bases teóricas y conceptuales y se propone evaluar las competencias genéricas y específicas; para desarrollar indicadores internacionalmente comparables" (Salganik, Rychen, Monsery y Stant, 1999, p: 7).

Estas pruebas SABER PRO antes llamadas Exámenes de Calidad de Educación Superior (ECAES) son reguladas por la Ley 1324 del 2009 en respuesta a lo expuesto en la Ley 3963 del 2009 donde los procesos de evaluación obtienen un carácter autocritico generando cambios de sus propias dinámicas, para responder a problemas contextualizados. Dentro de los cambios generados a partir de esta ley se destacan los realizados en 2011 en los programas de Ingeniería, Ciencias de la Educación y Ciencias Naturales, que comienzan a ser evaluados por competencias genéricas y competencias específicas (Ministerio de Educación Nacional, 2016).

Las competencias específicas se relacionan a los saberes concretos de determinado campo de conocimiento. Las primeras competencias específicas eran "seis (6) formuladas para las pruebas ECAES, se revisaron y ajustaron a las nuevas especificaciones técnicas del ICFES para concretarlas en las tres básicas y fundamentales" (MEN, 2016, p: 7); actualmente, las competencias específicas para los estudiantes de licenciatura en Colombia son: Enseñar; Formar, Evaluar (MEN, 2016).

Por otro lado, el debate en torno a la formación docente en donde problematizan asuntos como los conocimientos que componen al conocimiento profesional del profesor, las competencias específicas que orientan la formación docente y la pertinencia de algunas evaluaciones externas posibilita poner en evidencia las relaciones que entre estas se establecen.

En este sentido, el conocimiento profesional del profesor emerge como una temática de vital importancia en la presente investigación, pues este se compone de una serie de conocimientos subsidiarios diferenciadores frente a otros conocimientos profesionales relativos a otros campos de saber, dado que la profesión docente en términos de Tardif (2004), posee un objeto heterogéneo, conformado por sujetos e interacciones humanas (Valbuena, 2008). Shulman (1986) define siete (7) tipos de conocimiento que debe contener un profesor: Conocimiento del contenido temático de la materia o asignatura (CA), Pedagógico general curricular, Pedagógico del contenido (CPC), Conocimiento de los aprendices y sus características, Conocimiento del contexto educativo, Conocimiento de los fines, propósitos y valores educacionales y sus bases filosóficas e históricas (Arteta, Fonseca, lbáñez, Chona y Martínez, 2009).

Para Porlan (2003) el conocimiento del contenido es relevante puesto que la estructura conceptual es la que le permite al docente establecer relaciones y redes conceptuales amplias (Valbuena, 2008). Según la clasificación de Schwab (1978) citado en (Shulman, 1986) la composición del conocimiento del contenido abarca desde los con- 
Bio - grafía. Escritos sobre la Biología y su Enseñanza. ISSN 2027-1034

Edición Extraordinaria. p.p. 431- 440

Memorias del IX Encuentro Nacional de Experiencias en Enseñanza de la Biología y la Educación Ambiental. IV Congreso Nacional de Investigación en Enseñanza de la Biología.

ceptos y contenidos concretos a impartir hasta su organización sustantiva y sintáctica. Por un lado, a estructura sustantiva se refiere a "la variedad de formas como los conceptos y principios básicos son organizados para incorporar los hechos" (Shulman, 1986, p: 9, citado en Valbuena, 2008, p: 73). Marcelo (1999), propone que este conocimiento contiene al conocimiento de los marcos teóricos, tendencias y la estructura interna de determinada disciplina, trascendiendo así la simple observación de conceptos y principios (Valbuena, 2008). Por otro lado, la estructura sintáctica se refiere a los mecanismos de verificación y validación de los contenidos, "la sintaxis de la disciplina aporta las reglas para determinar cuál aseveración tiene mayor nivel de justificación" (Shulman, 1986, p: 9, citado en Valbuena, 2008, p: 73). Grossman (1990) afirma que esta estructura incluye a la estructura sustantiva además de relacionarla al manejo del docente sobre determinados criterios aceptados, necesarios para orientar la investigación científica y la validación de nuevos conocimientos. (Valbuena, 2008).

Algunas de las relaciones e incidencia en la enseñanza del conocimiento del contenido se traducen en procesos desarrollados por el docente, tales como: establecer relaciones entre diversos conceptos, realizar síntesis de los contenidos, identificar los principios que orientan la disciplina, diseñar prácticas de laboratorios por ejemplo, donde expone como un fenómeno se presenta en la cotidianidad para así formular hipótesis y preguntas relacionadas con objetivos de aprendizaje (Valbuena, 2008). De manera conjunta, se trasciende la idea de que solo se requieren los contenidos y la estructura de una disciplina, al identificar la importancia de reconocer elementos históricos y epistemológicos de esta, pues dotan al maestro de elementos útiles en la enseñanza como la identificación de conceptos estructurantes además de las dinámicas propias de la producción del conocimiento (Gagliardi, 1986, 1988; Gagliardi y Giordan, 1986; Gil-Pérez, 1993; Giordan y De Vecchi, 1999, citado en Valbuena 2008).

Teniendo en cuenta la estrecha relación que existe entre la evaluación y los contenidos específicos de determinada profesión, que para nuestro caso serán los docentes del área de Ciencias Naturales y Educación Ambiental se pretende determinar mediante análisis de contenido si el conocimiento del contenido interviene en la formulación de preguntas en la prueba SABER PRO.

\section{METODOLOGIA}

La metodología se desarrolla con base a las fases propuestas por Raigada (2002) donde identifica cinco (5) ineludibles en un análisis de contenido cualitativo:

- Determinación del objeto de análisis. El objeto de análisis puede definirse como la dificultad que no se puede resolver de forma mecánica, sino que requiere una investigación empírica o conceptual; en el análisis de contenido confluyen estos dos tipos de investigación (Abela, 2002). Teniendo en cuenta esto, definimos como objeto de análisis a la inclusión del CA en la formulación de las preguntas de la prueba SABER PRO.

- Selección de la comunicación que será estudiado: Se refiere a la selección de los elementos dentro de un texto que serán objeto de análisis; el diseño de categorías relevantes y la evaluación de los indicadores de selección que lo determinan (Raigada, 2002). Los documentos usados durante el desarrollo de esta fase son extraídos de las guías para los módulos: Enseñar, Evaluar y Formar encon- 
Bio - grafía. Escritos sobre la Biología y su Enseñanza. ISSN 2027-1034

Edición Extraordinaria. p.p. 431- 440

Memorias del IX Encuentro Nacional de Experiencias en Enseñanza de la Biología y la Educación Ambiental. IV Congreso Nacional de Investigación en Enseñanza de la Biología.

tradas en la base de datos del ICFES: http://www.icfes.gov.co/noticias/item/1982modulos-saber-pro.

- Selección de las categorías que se utilizarán: La generación de categorías será mediante el método inductivo el cual según (Strauss, 1987), citado por Rodríguez et al, (2005) se caracteriza por la elaboración de categorías desde la lectura y análisis del material recolectado sin tener en cuenta categorías propuestas previamente, esto por la dificultad de encontrar estudios que generen este tipo de categorías. Las categorías propuestas son: Conocimiento del contenido y otro tipo de conocimiento, en donde se incluye, simbolizando con una " $X$ " unidades de análisis para su posterior análisis.

- Selección de las unidades de análisis: Las unidades de análisis son los fragmentos de la comunicación que apoyan y se incluyen dentro de las categorías propuestas para explicar el fenómeno en cuestión Abela (2002). Para la presente investigación las unidades serán los desempeños propuestos a ser evaluados por el ICFES en la prueba SABER PRO. Se incluirán dentro de la categoría Conocimiento del contenido a las unidades de análisis que presenten la estructura sintáctica y sustantiva señalada por (Valbuena, 2008) necesaria para identificar el uso del CA, mientras que las que no tengan esta condición serán incluidas en la categoría de otro tipo de conocimiento.

- Selección del sistema de recuento o de medida: Para esta fase final se ha acudido a la triangulación de investigador en donde según Valencia (2013), se emplean múltiples observadores con el objetivo de darle robustez a los resultados cualitativos obtenidos. Esta triangulación es efectuada por los cuatro (4) investigadores de forma individual para evitar condicionamientos en el momento de incluir las unidades de análisis en sus respectivas categorías.

\section{RESULTADOS:}

La siguiente tabla muestra las competencias específicas para docentes: Evaluar, Enseñar y Formar, unidades de registro y las categorías propuestas para esta investigación.

Adicionalmente se muestran ejemplos tomados de dicho modulo que ejemplifican la forma en que deberían ser diseñadas estas preguntas

Tabla 1. Matriz de análisis

\begin{tabular}{|c|c|c|c|}
\hline \multirow{2}{*}{ Competencias } & Unidades de análisis & \multicolumn{2}{|c|}{ Categorías } \\
\cline { 2 - 4 } & Desempeño a evaluar & $\begin{array}{c}\text { Conocimiento } \\
\text { del contenido }\end{array}$ & $\begin{array}{c}\text { Otro tipo de } \\
\text { conocimiento }\end{array}$ \\
\hline EVALUAR & $\begin{array}{c}\text { Utilizar la evaluación para hacer } \\
\text { seguimiento a los procesos } \\
\text { educativos. }\end{array}$ & $\mathrm{X}$ \\
\hline
\end{tabular}


Bio - grafía. Escritos sobre la Biología y su Enseñanza. ISSN 2027-1034

Edición Extraordinaria. p.p. 431- 440

Memorias del IX Encuentro Nacional de Experiencias en Enseñanza de la Biología y la Educación Ambiental. IV Congreso Nacional de Investigación en Enseñanza de la Biología.

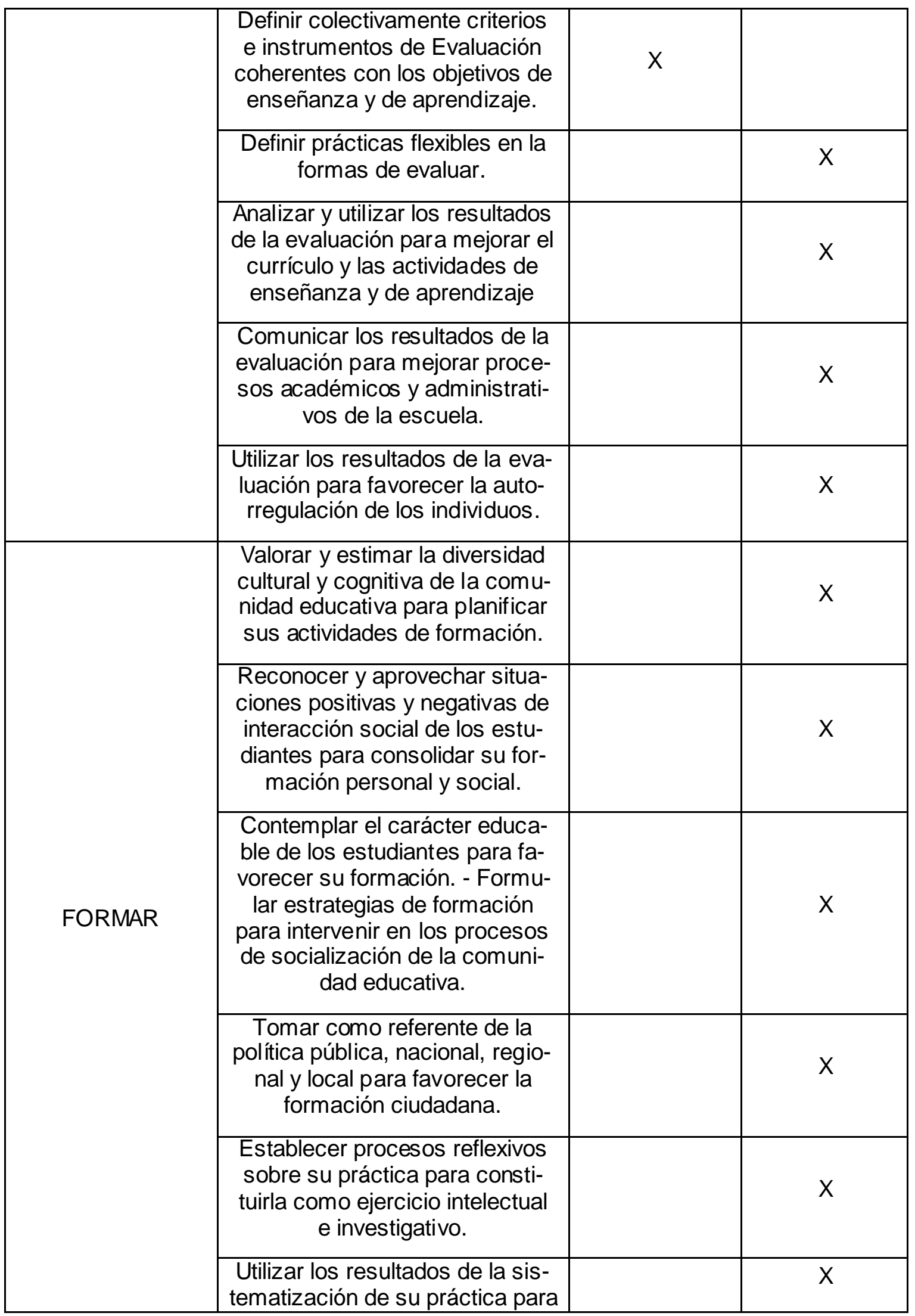


Bio - grafía. Escritos sobre la Biología y su Enseñanza. ISSN 2027-1034

Edición Extraordinaria. p.p. 431- 440

Memorias del IX Encuentro Nacional de Experiencias en Enseñanza de la Biología y la Educación Ambiental. IV Congreso Nacional de Investigación en Enseñanza de la Biología.

\begin{tabular}{|c|c|c|c|}
\hline & $\begin{array}{l}\text { diseñar estrategias para su cua- } \\
\text { lificación. }\end{array}$ & & \\
\hline & $\begin{array}{l}\text { Utilizar principios de la política } \\
\text { pública nacional, regional y local } \\
\text { para potenciar desarrollos de } \\
\text { las comunidades educativas. }\end{array}$ & & $X$ \\
\hline \multirow{6}{*}{ ENSEÑAR } & $\begin{array}{c}\text { Conocer la naturaleza de la } \\
\text { disciplina que enseña para re } \\
\text { contextualizarla en el acto edu- } \\
\text { cativo }\end{array}$ & $X$ & \\
\hline & $\begin{array}{c}\text { Conocer la didáctica de la disci- } \\
\text { plina que enseña para favorecer } \\
\text { los aprendizajes de los estu- } \\
\text { diantes. }\end{array}$ & & $X$ \\
\hline & $\begin{array}{l}\text { Establecer objetivos de ense- } \\
\text { ñanza para planear la clase. }\end{array}$ & $X$ & \\
\hline & $\begin{array}{l}\text { Diseñar mallas curriculares para } \\
\text { organizar secuencias de ense- } \\
\text { ñanza en el plano de estudios. }\end{array}$ & $X$ & \\
\hline & $\begin{array}{l}\text { Planificar estrategias de ense- } \\
\text { ñanza, aprendizaje y evalua- } \\
\text { ción. }\end{array}$ & & $X$ \\
\hline & $\begin{array}{c}\text { Relacionar las actitudes, prácti- } \\
\text { cas y experiencias de los estu- } \\
\text { diantes para el desarrollo de su } \\
\text { clase. }\end{array}$ & & $\mathrm{X}$ \\
\hline
\end{tabular}

A continuación en la tabla 2 se encuentran una serie de preguntas obtenidas desde la página web del ICFES. Estas preguntas son de opción múltiple.

Tabla 2. Ejemplos preguntas.

\begin{tabular}{|l|l|l|l|}
\hline Competencia & Pregunta orientadora & Respuesta & Desempeño \\
\hline
\end{tabular}


Bio - grafía. Escritos sobre la Biología y su Enseñanza. ISSN 2027-1034

Edición Extraordinaria. p.p. 431- 440

Memorias del IX Encuentro Nacional de Experiencias en Enseñanza de la Biología y la Educación Ambiental. IV Congreso Nacional de Investigación en Enseñanza de la Biología.

\begin{tabular}{|c|c|c|c|}
\hline EVALUAR & $\begin{array}{l}\text { Un profesor de español va a } \\
\text { trabajar una unidad sobre po- } \\
\text { esía y quiere que sus estudian- } \\
\text { tes aprendan sobre: } \\
\text { • Aspectos formales de } \\
\text { la poesía. } \\
\text { • Propósitos del género. } \\
\text { • Significado de las figu- } \\
\text { ras literarias utilizadas. } \\
\text { El mejor ejercicio de evalua- } \\
\text { ción, coherente con los objeti- } \\
\text { vos sería: }\end{array}$ & $\begin{array}{l}\text { Contrastar } \\
\text { un poema y } \\
\text { un ensayo } \\
\text { dando cuenta } \\
\text { de las figuras } \\
\text { literarias, la } \\
\text { estructura y } \\
\text { los propósi- } \\
\text { tos. }\end{array}$ & $\begin{array}{l}\text { Definir colecti- } \\
\text { vamente crite- } \\
\text { rios e instru- } \\
\text { mentos de } \\
\text { Evaluación } \\
\text { coherentes con } \\
\text { los objetivos de } \\
\text { enseñanza y } \\
\text { de aprendizaje. }\end{array}$ \\
\hline FORMAR & $\begin{array}{l}\text { Los medios de comunicación } \\
\text { masiva se han constituido en } \\
\text { importante competidor de la } \\
\text { escuela en la transmisión de } \\
\text { valores sociales y morales. } \\
\text { Frente a esta problemática, se } \\
\text { puede concluir que correspon- } \\
\text { de a la institución educativa. }\end{array}$ & $\begin{array}{l}\text { Aprovechen } \\
\text { internet para } \\
\text { la adquisi- } \\
\text { ción de in- } \\
\text { formación } \\
\text { sobre los } \\
\text { temas am- } \\
\text { bientales }\end{array}$ & $\begin{array}{l}\text { Valorar y esti- } \\
\text { mar la diversi- } \\
\text { dad cultural y } \\
\text { cognitiva de la } \\
\text { comunidad } \\
\text { educativa para } \\
\text { planificar sus } \\
\text { actividades de } \\
\text { formación. }\end{array}$ \\
\hline ENSEÑAR & $\begin{array}{l}\text { Teniendo en cuenta que los } \\
\text { límites entre las disciplinas son } \\
\text { cada vez más tenues, las com- } \\
\text { petencias transversales están } \\
\text { adquiriendo mayor presencia y } \\
\text { valor en los procesos de for- } \\
\text { mación integral. Su incorpora- } \\
\text { ción en los procesos educati- } \\
\text { vos requiere cambios en la } \\
\text { planeación, la selección de } \\
\text { metodologías y la evaluación. } \\
\text { Por no ser específicas de un } \\
\text { campo social, su integración } \\
\text { curricular requiere. }\end{array}$ & $\begin{array}{l}\text { La definición } \\
\text { de los atribu- } \\
\text { tos para dar } \\
\text { cuenta de su } \\
\text { apropiación }\end{array}$ & $\begin{array}{c}\text { Conocer la } \\
\text { naturaleza de } \\
\text { la disciplina } \\
\text { que enseña } \\
\text { para re contex- } \\
\text { tualizarla en el } \\
\text { acto educativo }\end{array}$ \\
\hline
\end{tabular}

\section{ANALISIS:}

La tabla 1 muestra como la competencia evaluar contiene el desempeño 2 en relación con el Conocimiento del contenido, puesto que el docente para plantear los objetivos de enseñanza aprendizaje debe conocer el contenido para cada institución además de los propuestos por los lineamientos curriculares del Ministerio de Educación Nacional. Esta competencia busca que el docente reflexione, haga seguimiento y 
Bio - grafía. Escritos sobre la Biología y su Enseñanza. ISSN 2027-1034

Edición Extraordinaria. p.p. 431- 440

Memorias del IX Encuentro Nacional de Experiencias en Enseñanza de la Biología y la Educación Ambiental. IV Congreso Nacional de Investigación en Enseñanza de la Biología.

tome decisiones sobre el proceso de formación, esto para generar una autorregulación y mejorar el ejercicio docente (MEN, 2016), sin embargo, para esta competencia en los otros cinco (5) desempeños a evaluar se deja de lado la estructura sustantiva y sintáctica presente en el conocimiento del contenido lo cual afecta también la verificación y la validación del contenido de disciplinas específicas.

La competencia formar no se ve influenciada por el uso del Conocimiento del contenido, puesto que esta se ve orientada a la construcción social que sin evidenciar por lo menos de manera la formación disciplinar, así pues se busca que el docente re conceptualice y utilice conocimientos que le permitan crear ambientes educativos en los cuales los estudiantes, el profesor y de la comunidad puedan participar en el proceso educativo (MEN, 2016).

Para la competencia enseñar, se busca generar preguntas que evalúen, la comprensión, la formulación además del uso de la didáctica de las disciplinas con el propósito de favorecer aprendizajes en los estudiantes (MEN, 2016). Al igual que en la competencia evaluar, vemos como el uso del Conocimiento del contenido se ve con un bajo porcentaje respecto a los demás saberes que debe poseer un docente, esto representado en los desempeños un (1), tres (3) y cuatro (4), donde nuevamente para planear proyectos curriculares, planes de estudio y unidades de aprendizaje es necesario tener conocimientos concretos con estructuras sintácticas y sustantivas fuertes y actualizados.

La baja inclusión del conocimiento del contenido y su correspondiente estructura sustantiva y sintáctica (Valbuena, 2008) se generan preguntas que no reconocen las especificidades propias de cada disciplina. En efecto, si revisamos, por ejemplo, la pregunta orientadora para la competencia evaluar, nos daremos cuenta que aunque se hace referencia a un Conocimiento del contenido este pertenece al campo de la enseñanza de la Literatura lo cual al momento de ser resuelto por Licenciados de las áreas de Ciencias Naturales y Educación Ambiental generara sesgos o errores en su posteriormente calificación. Algo similar ocurre con la pregunta orientadora para la competencia enseñar, la cual intenta generar procesos de cambio y dinamismo de las propias herramientas y costumbres evaluativas con base en indagaciones sobre el manejo que el docente hace de los diferentes saberes de cada disciplina, sin embargo explícitamente habla de una in especificidad en esta competencia. En cuanto a la pregunta orientadora para la competencia formar y como vimos en el análisis de los desempeños, para esta competencia el conocimiento del contenido en Ciencias Naturales se encuentra ausente.

\section{CONCLUSIONES:}

Las competencias específicas que se evalúan en la prueba SABER PRO del ICFES, si bien tratan de categorizar dichas evaluaciones de acuerdo a los diferentes profesionales en formación, para el caso de la docencia no abarca la totalidad de las competencias para las que se está preparando, dejando ausente las particularidades de la enseñanza de las Ciencias Naturales y la Educación Ambiental.

En las competencias enseñar y evaluar a pesar de hacer explícito el saber propio de cada disciplina no hay un verdadero reconocimiento del conocimiento del contenido pues se usa indiferenciadamente el contenido tanto de disciplinas Humanísticas- 
Bio - grafía. Escritos sobre la Biología y su Enseñanza. ISSN 2027-1034

Edición Extraordinaria. p.p. 431- 440

Memorias del IX Encuentro Nacional de Experiencias en Enseñanza de la Biología y la Educación Ambiental. IV Congreso Nacional de Investigación en Enseñanza de la Biología.

Sociales junto con las disciplinas que estudian la las Ciencias Naturales. Prescindir de las estructuras sustantiva y sintáctica a la hora de formular preguntas para medir el alcance de competencias específicas para el profesorado en formación (de distintas áreas tales como biología, matemáticas, ciencias sociales entre otras) puede generar sesgos en el análisis que realizan instituciones como el ICFES sobre dichas competencias, puestos que el conocimiento del contenido es un elemento de altísimo valor en la composición del conocimiento profesional del profesor.

\section{REFERENCIAS:}

Abela, J. A. (2002). Las técnicas de análisis de contenido: una revisión actualizada

Arteta, J., Fonseca, G., Ibáñez S., Chona, G., \& Martínez, S. (2009). El conocimiento profesional del profesor y el desarrollo de competencias científicas investigativas. Barcelona.

Grossman, P. L. (1990). The making of a teacher: Teacher knowledge and teacher education. Teachers College Press.

Ley 1324. Ministerio de Educación Nacional, Bogotá, Colombia, 2009.

Ley 3963. Ministerio de Educación Nacional, Bogotá, Colombia, 2009.

Marcelo García, C. (1999). Estudio sobre estrategias de inserción profesional en Europa. (pp. 101-143). Revista Iberoamericana de Educación.

Ministerio de Educación Nacional, (2016). LINEAMIENTOS DE CALIDAD PARA LAS LICENCIATURAS EN EDUCACIÓN (Programas de Formación Inicial de Maestros). (pp.1 - 37) Bogotá.

Porlán Ariza, R. (2003). Principios para la formación del profesorado de secundaria. Revista Interuniversitaria de formación del profesorado, 17(1).

Raigada, J. L. P. (2002). Epistemología, metodología y técnicas del análisis de contenido. Sociolinguistic Studies, 3(1), 1-42.

Rey, B. (1996). Las competencias transversales en cuestión. Paris. ESF. Traducción de Alejandro Madrid Zan. En:

http://www.philosophia.cl/biblioteca/Rey/Competencias\%20transversales.pdf

Rodríguez Sabiote, C., Lorenzo Quiles, O., \& Herrera Torres, L. (2005). Teoría y práctica del análisis de datos cualitativos. Proceso general y criterios de calidad. Revista Internacional de Ciencias Sociales y Humanidades. 15(2). SOCIOTAM.

Salganik. L., Rychen, D. Monsery, U y Stant, J (1999). Definición y Selección de

Competencias. Proyecto sobre competencias en el contexto de la OCDE. Análisis de base teórica y conceptual. Suiza. Oficina Federal de Estadística. OFE.

Shulman, L. S. (1986). Those who understand: Knowledge growth in teaching. Educational researcher, 15(2), 4-14. 
Bio - grafía. Escritos sobre la Biología y su Enseñanza. ISSN 2027-1034

Edición Extraordinaria. p.p. 431- 440

Memorias del IX Encuentro Nacional de Experiencias en Enseñanza de la Biología y la Educación Ambiental. IV Congreso Nacional de Investigación en Enseñanza de la Biología.

Tardif, M. (2004). Los saberes del docente y su desarrollo profesional. Narcea Ediciones.

Valbuena Ussa, E. O. (2008). El conocimiento didáctico del contenido biológico: estudio de las concepciones disciplinares y didácticas de futuros docentes de la Univers idad Pedagógica Nacional (Colombia). España. Universidad Complutense de Madrid.

Valencia, M. M. A. (2013). La triangulación metodológica: sus principios, alcances y limitaciones. Investigación y educación en enfermería, 18(1). 